\title{
Erratum to: Expression pattern of hTERT telomerase subunit gene in different stages of chronic myeloid leukemia
}

\author{
Ali Amini ${ }^{1}$ - Seyed Hamidollah Ghaffari ${ }^{2}$ - Yousef Mortazavi ${ }^{3}$ Karim Daliri ${ }^{4}$. \\ Shahrouz Taranejoo ${ }^{5} \cdot$ Kamran Alimoghadam $^{2} \cdot$ Ardeshir Ghavamzadeh $^{2}$
}

Published online: 29 February 2016

(C) Springer Science+Business Media Dordrecht 2016

\section{Erratum to: Mol Biol Rep (2014) 41:5557-5561 \\ DOI 10.1007/s11033-014-3472-3}

In the original publication, surname of author Yousef Mortazai was published incorrectly and is now corrected as Yousef Mortazavi.

The online version of the original article can be found under doi:10.1007/s11033-014-3472-3.

Yousef Mortazavi

kinlabgen@yahoo.com

1 Department of Biomedicine Allied Medical School, Shahid Sadoughi University of Medical Sciences, Yazd, Iran

2 Hematology-Oncology and BMT Research Center, Shariaty Hospital, Tehran University of Medical Sciences, Tehran, Iran

3 Department of Pathology and Molecular Medicine, Zanjan University of Medical Sciences, Zanjan, Iran

4 Department of Medical Genetics, Medical School, Shiraz University of Medical Sciences, Shiraz, Iran

5 Department of Chemical Engineering, Monash University, Melbourne, Australia 